\title{
Comparison of Inhibitory Effects of 17-AAG Nanoparticles and Free 17-AAG on HSP90 Gene Expression in Breast Cancer
}

\author{
Masoud Gandomkar Ghalhar', Abolfazl Akbarzadeh ${ }^{3}$, Mohammad Rahmati ${ }^{2}$, \\ Hassan Mellatyar ${ }^{1}$, Hassan Dariushnejad ${ }^{1}$, Nosratallah Zarghami ${ }^{1,24 *}$, Amin \\ Barkhordari $^{1}$
}

\begin{abstract}
Background: HSP90 may be overexpressed in cancer cells which are greatly dependent on Hsp90 function. Geldanamycin derivative 17 allylamino-17-demethoxygeldanamycin (17-AAG) inhibits the function and expression of HSP90. 17-AAG has poor water-solubility which is a potential problem for clinical practice. In this study for improving the stability and solubility of molecules in drug delivery systems we used a $\beta$-cyclodextrin17AAG complex. Materials and Methods: To assess cytotoxic effects of $\beta$-cyclodextrin-17AAG complexes and free 17AAG, colorimetric cell viability (MTT) assays were performed. Cells were treated with equal concentrations of $\beta$-cyclodextrin- 17AAG complex and free 17AAG and Hsp90 gene expression levels in the two groups was compared by real-time PCR. Results: MTT assay confirmed that $\beta$-cyclodextrin- 17AAG complex enhanced 17AAG cytotoxicity and drug delivery in T47D breast cancer cells. The level of $\mathrm{Hsp90}$ gene expression in cells treated with $\beta$-cyclodextrin- 17AAG complex was lower than that of cells treated with free 17AAG $(P=0.001)$. Conclusions: The results demonstrated that $\beta$-cyclodextrin- 17AAG complexes are more effective than free 17AAG in down-regulating HSP90 expression due to enhanced $\beta$-cyclodextrin-17AAG uptake by cells. Therefore, $\beta$-cyclodextrin could be superior carrier for this kind of hydrophobic agent.
\end{abstract}

Keywords: $\beta$-cyclodextrin - geldanamycin - cytotoxic effects - MTT assay - uptake

Asian Pac J Cancer Prev, 15 (17), 7113-7118

\section{Introduction}

Breast tissue malignancy is the most common cause of cancer with a high mortality rate in women (Najafi et al., 2013). While the progress has decreased death rates of breast cancer, the complexity of breast cancer and several genetic abnormalities has made it difficult. therefore targeting a single pathway for inhibiting the activity of one element is improbable to be effective (Zajac et al., 2010; Ge et al., 2012; Shawky et al., 2014). Identification of a molecular target that will modulate mechanisms of several signaling pathways would be suitable for anticancer therap (Zhang et al., 2012; Cihan 2014). Heat shock protein 90 (HSP90) is believed to be an excellent molecular target in cancer treatment. HSP90 overexpression has been found in cancer cells and showed these cells are vastly dependent on the Hsp90 function(Shirinbayan and Roshan 2011). HSP90 is a molecular chaperone that is induced in response to cellular stress and stabilizes client proteins involved in cell cycle control and proliferative/anti apoptotic pathways (Richardson et al., 2011; Sakthivel et al., 2012; Dobo et al., 2013; Wu et al., 2014).

This molecule is necessary for stability of several oncogenic client proteins (ERBB2, B-RAF, CDK4, AKT, mutant p53, among others) involved in transcriptional regulation, signal transduction, and cell cycle control as well as in other critical processes leading to malignant phenotype (Zajac et al., 2010). Therefore, Hsp90 plays a unique role in cellular homeostasis, thus has been an encouraging anticancer target. In vivo, HSP90 itself exists as a homodimer and each monomer chain contains of 732 amino acids, and comprises an N-terminal domain, a flexible linker region, a middle domain, and a $\mathrm{C}$-terminal domain. The N-terminal domain includes a nucleotidebinding site and also involved in interactions with cochaperones (Richardson et al., 2011). It is assumed that hydrolysis of ATP to ADP, at the nucleotide-binding site energizes the functions of the HSP90 chaperone complex (Bagatell and Whitesell 2004). Several inhibitors of HSP90 have been recognized; the most well categorized ones are derivatives of the benzoquinone ansamycin antibiotic geldanamycin (GA) and the macrolide

${ }^{1}$ Department of Medical Biotechnology, ${ }^{3}$ Department of Medical Nanotechnology, Faculty of Advanced Medical Sciences, ${ }^{2}$ Department of Clinical Biochemistry, Faculty of Medicine, ${ }^{4}$ Hematology and Oncology Research Center, Tabriz University of Medical Sciences, Tabriz, Iran*For correspondence: zarghami@tbzmed.ac.ir 
antibiotic radicicol that either bind to the $\mathrm{N}$-terminal domain, nucleotide binding site, inhibit the ATPase activity, ADP-ATP exchange activity, increase the usage of ubiquitin ligases to the HSP90 chaperone complex and consequently leading to increased degradation of client proteins by the proteasome pathway (Fukuyo et al., 2010).Geldanamycin derivative17-allylamino-17demethoxy-geldanamycin (17-AAG) exhibited lower toxicity and improved stability and demonstrated that 17-AAG induces reduction of key regulators of signal transduction in many human tumors, including colon and breast cancer (Usmani and Chiosis 2011; Schulz et al., 2012). In cancer cells, 17AAG binds more strongly to Hsp90, because it is in the form of a heteroprotein complex and in normal cells is mainly homodimeric and this could result in the selective accumulation of it in cancer cells (Usmani and Chiosis 2011). 17AAG has poor water-solubility which is a potential problem for clinical construction and obstacle for its clinical application (Guo et al., 2008). Despite all of these, Cyclodextrin is commonly used for improving the stability and solubility of molecules in drug delivery systems. There are three common types of Cyclodextrin including $\alpha$-Cyclodextrin, $\beta$-Cyclodextrin and $\gamma$-Cyclodextrin. The difference between these three types is in their internal cavity. The inner cavity of $\alpha$-Cyclodextrin is very tiny for $17 \mathrm{AAG}$ loading while the inner cavity of $\gamma$-Cyclodextrin is too large, but inner cavity of $\beta$-Cyclodextrin is suitable. $\beta$-Cyclodextrin is a semi-natural compound with low toxicity, which could enhance drug bioavailability (Challa et al., 2005; Yadav et al., 2014). $\beta$-Cyclodextrin was used for encapsulation of 17AAG (Sakthivel et al., 2012). In this study, we investigate that $\beta$-Cyclodextrin- 17AAG complex has inhibitory effect on Hsp90 gene expression in T47D breast cancer cell line, as well inhibitory function on hsp90 activity. Anticancer effect of free 17AAG and $\beta$-Cyclodextrin- 17AAG was compared. The level of Hsp90 gene expression after $24 \mathrm{~h}$ exposure was measured by Real-time PCR.

\section{Materials and Methods}

Fetal Bovine Serum (FBS), RPMI 1640, TripsinEDTA Antibiotics, and TRIzol reagent were purchased from Invitrogen (Germany). Syber Green Real Time PCR Master Mix kit was purchased from Roche (Germany). 3-(4,5-Dimethylthiazol-2-yl)-2,5-diphenyltetrazolium bromide (MTT), B-Cyclodextrin and 17AAG were purchased from Sigma (USA). T47D epithelial like breast cancer, prepared from Pasteur Institute cell bank of Iran, code: C203.

\section{Cell culture and cell line}

T47D epithelial like breast cancer cells were cultured in RPMI1640 complemented with $10 \%$ heat-inactivated fetal bovine serum (FBS), $0.05 \mathrm{mg} / \mathrm{ml}$ penicillin $\mathrm{G}$, $0.08 \mathrm{mg} / \mathrm{ml}$ streptomycin (Merck co, Germany), $2 \mathrm{mg} / \mathrm{ml}$ sodium bicarbonate and Cells were grown at $37^{\circ} \mathrm{C}$ in an incubator with $55 \%$ humidity and $5 \% \mathrm{CO}_{2}$.
Preparation of $\beta$-cyclodextrin-17AAG complex

$40 \mathrm{mg}$ of $\beta$-Cyclodextrin was dissolved in $8 \mathrm{~mL}$ deionized water; and $12 \mathrm{mg}$ of 17AAG was dissolved in $500 \mu$ lacetone. These two solutions were mixed together and were sited on the stirrer at $400 \mathrm{rpm}$ for $24 \mathrm{~h}$ to evaporate the acetone without a cap. Then, it was centrifuged at 1000 rpm for 5 min and supernatant were collected by freeze drying. (Yallapu et al., 2012; Yin et al., 2013)

\section{Size Distribution (SEM)}

The surface morphology of the nanospheres during the incubation time was observed by SEM. The nanographs of $\beta$-CD-17AAG in nanoparticles are shown in Figure (3). As it is demonstrated the size of the particles is about 30- $65 \mathrm{~nm}$ and dispersion of the particles was significantly improved. Also, the samples were layered with gold particles.

\section{Determination of $17 A A G$ loading}

$2 \mathrm{mg}$ of $\beta$-Cyclodextrin-17AAG complex was dissolved in 100mL dimethylsulfoxide (DMSO) and the solution was placed on shaker for $24 \mathrm{~h}$ at room temperature. Then, the solution was Centrifuged at 12,000 rpm and supernatant collected for the calculations. The standard 17AAGve of 17AAG concentration in DMSO was drowned using UV-Vis spectrophotometer by absorbance rate of $17 \mathrm{AAG}$.

\section{In vitro cytotoxicity (MTT assay)}

After growing, sufficient amount of cells in exponential phase of growth, exposed to free 17-AAG and $\beta$-Cyclodextrin-17AAG complex. Their Cytotoxic effects were measured by 24, 48 and 72h MTT assay in the triplicate model. Generally 2000 cell per well were cultured in a 96 well plate and after $24 \mathrm{~h}$ incubation, cells were treated with different concentrations of $\beta$ Cyclodextrin-17AAG complex $(0 \mu \mathrm{g} / \mathrm{ml}-320 \mu \mathrm{g} / \mathrm{ml})$ and free $17-\mathrm{AAG}(0 \mu \mathrm{g} / \mathrm{ml}-320 \mu \mathrm{g} / \mathrm{ml})$. In addition, $\beta$-Cyclodextrin in PBS or DMSO was used as the control. After this different exposure duration, medium was removed and then the cells were fed with $200 \mu \mathrm{l}$ fresh medium. Cells waited for $24 \mathrm{~h}$, next $50 \mu \mathrm{l}$ of $2 \mathrm{mg} / \mathrm{ml}$ MTT was dissolved in PBS and was added to each well and plate was covered with aluminum foil and incubated for $4 \mathrm{~h}$ in dark place. In the next step, wells content were removed and $200 \mu \mathrm{l}$ pure DMSO and $25 \mu \mathrm{l}$ Sorensen's glycine buffer were added to wells. Finally, total of formazan was determined by measuring the absorbance at $570 \mathrm{~nm}$ by ELISA plate reader (Bio Tech Instruments, USA) with a reference wavelength of $630 \mathrm{~nm}$.

\section{Cell treatment}

After appointment of IC50, a counted (106) cell was treated in a 6-well plate with different concentrations of free 17-AAG and $\beta$-Cyclodextrin-17AAG complex $(0$, $10,20,40,80,160$ and $320 \mu \mathrm{g} / \mathrm{ml})$. For control cells, the same volume of $10 \%$ DMSO without drugs was added to plate containing the control cells. An equivalent amount of $\beta$-Cyclodextrin in PBS was used as another control. Next, plates were incubated for 24,48 and $72 \mathrm{~h}$ exposure 
time in an incubator $5 \% \mathrm{CO}_{2}$ and $37^{\circ} \mathrm{C}$.

\section{RNA extraction and reverse transcription}

Total RNA was extracted with Trizol in a clean RNasefree tube, according to the manufacturer's protocol for cell lines. Efficiency of our extraction tested with nanodrop analysis. Also purity of our extracted RNA was calculated with spectrophotometer at $260 / 280 \mathrm{~nm}$ ratio and the firmness of extracted RNA was defined by electrophoresis in $0.5 \mu \mathrm{g} / \mathrm{ml}$ ethidium bromide contained agarose gels. Complementary DNA (cDNA) was synthesized using random hexamer primers with purchased reverse transcriptase kit from fermentas, K1622.

\section{Quantitative real-time PCR assay}

Quantity of hsp90 gene expression was determined by quantitative Real-time PCR technique using Syber Green-I by means of the Rotor-GeneTM 6000 machinery (Corbett research, Australia) according to the manufacturer's protocols. For real-time PCR, hsp90 primers (Genbank accession: NM_005348,bp60-79) and beta actin primers (Genbank accession: NM-001101, bp 787-917) were used. These primers were blasted by primer- blast site on NCBI website. The forward $(\mathrm{F})$ and reverse $(\mathrm{R})$ primer sequences of hsp90 and $\beta$-actin used in real-time PCR were shown in Table (1). For hsp90, a 162bp amplicon and for beta actin a $131 \mathrm{bp}$ amplicon were generated in a $25 \mu \mathrm{l}$ reaction mixture that contained: 5 pmole of the forward and reverse PCR primers of beta actin or for hsp90, 2X PCR Master Mix Syber Green I and $2 \mu l$ of the cDNA. The Beta-Actin mRNA was calculated as the internal standard control gene by specific primers. The program for real-time PCR reaction contained of an Inhibition of hsp90 Gene Expression by $\beta$-Cyclodextrin-17AAG initial denaturation step at $95^{\circ} \mathrm{C}$ for $5 \mathrm{~min}$ and 45 cycles initial denaturation step at $95^{\circ} \mathrm{C}$ for $5 \mathrm{~min}$ and 45 cycles of denaturation ( $95^{\circ} \mathrm{C}$ for 10 seconds), annealing $\left(60^{\circ} \mathrm{C}\right.$ for 10 seconds), and extension $\left(72^{\circ} \mathrm{C}\right.$ for 25 seconds). Finally, amplicons were experienced with melting 17AAGve analysis of 95- $65^{\circ} \mathrm{C}$.Changes that oc $17 \mathrm{AAGred}$ in hsp90 expression amounts between the control and T47D cells that treated with $\beta$-Cyclodextrin 17AAG complex and free 17-AAG, normalized to $\beta$-Actin mRNA amounts, calculated with the $2^{-\Delta \Delta C T}$ method. Each DNA sample was divided so that hsp90 and beta actin could be amplified, in parallel, the reactions were done in duplicate with equal amounts of starting material.

\section{Statistical analysis}

Statistical analyses were performed with GraphPad Prism 6.01 software. Results were expressed as the mean \pm standard deviation (SD). Statistical differences were assessed by unpaired student t-test; and a value of $\mathrm{P}$ less than 0.05 was considered significant.

\section{Results}

\section{Determination of 17AAG loading}

Standard curve of 17AAG concentration in DMSO was prepared via UV-Vis spectrophotometer at $450 \mathrm{~nm}$. One mg of $\beta$-Cyclodextrin-17AAG complex contained $575.69 \mu \mathrm{g} 17 \mathrm{AAG}$.

Table 1. Primer Sequences

\begin{tabular}{llcll}
\hline Oligonucleotide & & Location & Sequence & PCR product size \\
\hline Hsp90 $\alpha$ & Forward primer & 60 & 5'AGGCTTCTGGAAAAAGCGCC3' & \\
& Reverse primer & 221 & 5'GTTGGTCTTGGGTCTGGGTT3' & 162bp \\
Beta-actin & Forward primer & 787 & 5'TCCCTGGAGAAGAGCTACG3' & 131bp \\
& Reverse primer & 917 & 5'GTAGTTTCGTGGATGCCACA3' & (3) \\
\hline
\end{tabular}

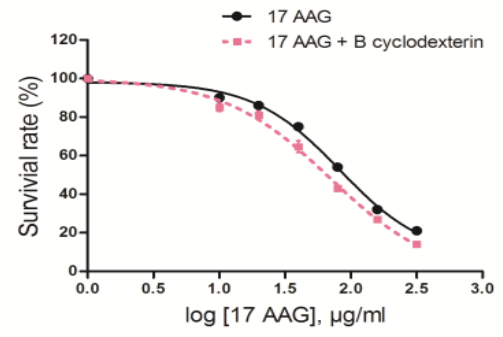

IC50 24

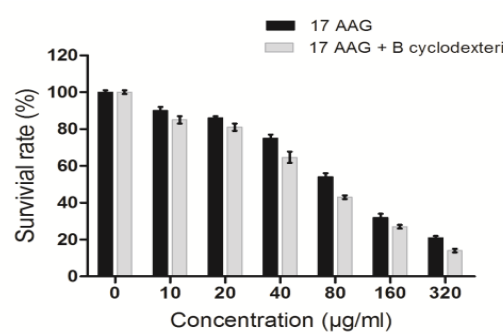

MTT 24

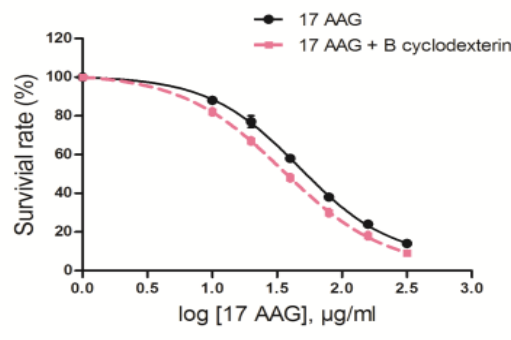

IC50 48

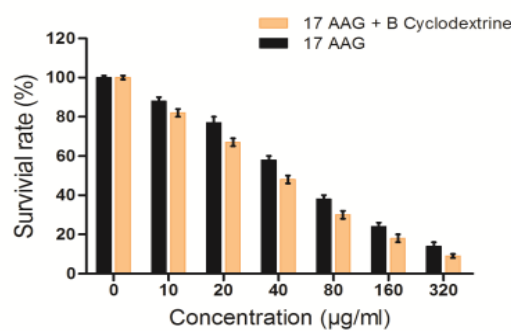

MTT 48

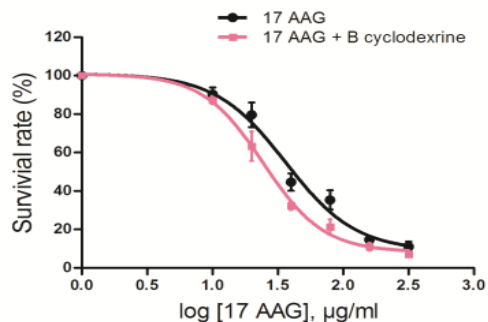

IC50 72

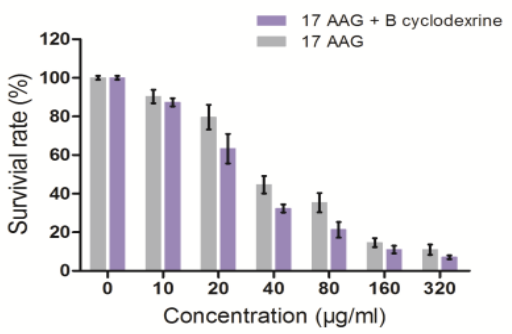

MTT 72

Figure 1. Cytotoxicity Effect of $\beta$-Cyclodextrin- 17AAGComplex and free 17AAG on T47D for A) 24h ; B) 48h ; C) 72h Exposure 


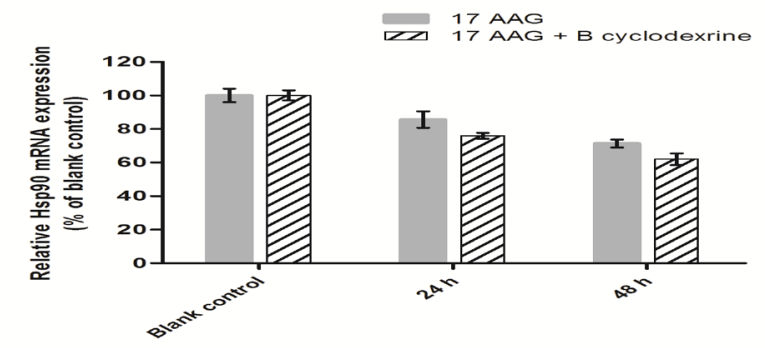

Figure 2. Level of HSP90 mRNA Expression in Cells Treated with CD-17AAG or Free 17AAG

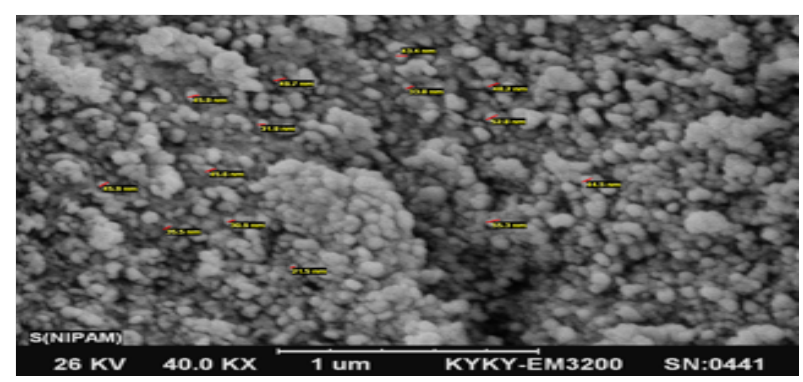

Figure 3. The Surface Morphology of the Nanospheres During the Incubation Time

Effects on cell viability

In this study to evaluate the cytotoxic effect (MTT assay) of $\beta$-CD-17AAG complex and free $17 \mathrm{AAG}$, T47D breast cancer cell lines were treated with different concentration mentioned above. The obtained IC50 of free $17 \mathrm{AAG}$ for 24,48 and $72 \mathrm{~h}$ was 82,46 and $35 \mu \mathrm{g} / \mathrm{m}$ and $\beta$-CD-17AAG complex for 24,48 and $72 \mathrm{~h}$ was 69,35 and $24 \mu \mathrm{g} / \mathrm{m}$ respectively. Our Data analysis of cytotoxicity assay showed that IC50 of $\beta$-Cyclodextrin-17AAG complex on T47D breast cancer cell line was time and dose-dependent (Figure 1).

\section{Effect on gene expression}

Cells were treated with $\beta$-CD-17AAG complex and free 17AAG for 24 and $48 \mathrm{~h}$. The expression of hsp90 mRNA levels was calculated via q-RT PCR. The level of hsp90 mRNA was standardized to mRNA level of the uniformly expressed housekeeping gene, beta actin, within each sample. Increasing $2^{-\Delta \Delta \mathrm{Ct}}$ amount resulted in enhanced expression of mRNA levels. Data analysis of q-RT PCR exhibited that with increasing concentration of $\beta$-Cyclodextrin17AAG complex, a decreasing trend appeared in mRNA level of hsp90. Each sample was repeated two times. Q-RT PCR results showed a considerable decrease in hsp90 gene expression in the treated cells in comparison with the control cells. Compared to $17 \mathrm{AAG}$, in the same concentration, $\beta-\mathrm{CD}$ 17AAG resulted in a lower level and expression of hsp90 mRNA. When we treated T47D cells with 61 and $41 \mu \mathrm{g} / \mathrm{m}$ concentrations of $\beta$-Cyclodextrin-17AAG complex for 24 and 48 hours, expression of hsp90 was significantly reduced (Figure2).

\section{Discussion}

While Chemotherapy has toxic side effects in healthy tissues in treatment of human cancers, Nanotechnology attempts to resolve these problems by encapsulating or loading drugs to nonmaterial which are resistant to drug efflux (Tsuda, 2010; Ghasemali et al., 2013). In recent times, Cyclodextrin nanoparticles are used mostly as recyclable transporters for drug delivery and several studies have shown that encapsulating drugs to Cyclodextrin polymers decreases adverse side effects of the drugs leading to use lower dosages of drug (Challa et al., 2005). In recent years, targeting Hsp90 has appeared as an exciting therapeutic intervention for a wide variety of human cancers. Hsp90 play a critical role in maturation and function of client proteins which related to oncogenic pathways, and expression levels of it seem to be upregulated in cancer (Challa et al., 2005). A variety of Hsp90 inhibitors have emerged as promising anticancer agents. 17AAG decreases hsp90 activity by inhibition of nucleotide binding site in breast cancer cells (Karkoulis et al., 2010). Through its ability to control the activity and stability of many client proteins involved in the oncogenic process, targeting Hsp90 has the potential to affect all the hallmarks of cancer (Zajac et al., 2010). It is unclear whether cell stress is important for efficiency of HSP90 inhibitors (Pick et al., 2007). 17AAG has poor water-solubility which is a potential problem for clinical construction and obstacle for its clinical application (Guo et al., 2008). However, Cyclodextrin is commonly used for improving the stability and solubility of molecules in drug delivery systems. In the present study, we have comparatively studied the antiproliferative effects of free $17-\mathrm{AAG}$ and $\beta$-CD-17AAG complex in breast cancer cells as shown in figure (1 and 2). we see Free 17-AAG compared with $\beta$-CD-17AAG complex induced Hsp90 downregulation in breast cancer cell lines showing the efficiency of our nanoparticle by comparison between our results obtained from T47D cell lines treated with $\beta$-Cyclodextrin-17AAG-complex and free 17AAG over a 24,48 and 72 hours treatment period. As shown in pervious study, treatment with $17-\mathrm{AAG}$ declined the levels of the growth promoting client protein kinases ,transcription factors (Karkoulis et al., 2010; Zajac et al., 2010) and it maybe a result of the fact that $\beta$-Cyclodextrin17AAG complex nano- particles reduce hsp90 mRNA gene expression especially when its concentration is increased. It should be noted that exposure dose also plays a key role in the inhibition of expression levels (a time-and dose-dependent manner similar to that of the cell growth inhibition). Hsp90 inhibitors are being actively considered as potential anti-tumor agents, because hp90 is in the form of a heteroprotein complex unlike in normal cells that is mainly in homodimeric shape. This could cause the selective accumulation of these molecules in cancer cells which results in a highly specific treatment with fewer side effects (Guo et al., 2008). Our experiments demonstrated that when we treat cell lines with the same values of $\beta$-Cyclodextrin-17AAG complex and 17AAG-free, under the same conditions in the three exposure times 24,48 and $72 \mathrm{~h}, \beta$-Cyclodextrin$17 \mathrm{AAG}$ complex is more effective and kill some more breast cancer cells. For evidence, IC50s are different in the stated exposure times and decrease with time as shown in the Figure (1). In conclusion, our data shows that $\beta$-Cyclodextrin- 17AAG complex had inhibitory 
effect on breast cancer T47D cell line. This inhibition was time and dose-dependent(Bhattacharjee et al., 2012). Cytotoxic effect of $\beta$-Cyclodextrin- 17AAG complex in the cells was increased with increasing concentration of $\beta$-Cyclodextrin-17AAG complex. Data analysis showed that by increasing concentration of $\beta$-Cyclodextrin17AAG complex, decreasing trend of hsp90 expression was observed. In summary, our results showed that low dosage of $\beta$-Cyclodextrin-17AAG complex has more inhibitory effect on expression of hsp90 mRNA than 17AAG free. Besides, $\beta$-Cyclodextrin-17AAG complex has fewer side effects than 17AAG free and has more inhibitory effect on hsp90 expression and function; consequently we can use this complex $(\beta$-Cyclodextrin$17 \mathrm{AAG}$ ) as a new anticancer compound in breast cancer treatment (Valizadeh et al., 2012; Mirakabad et al., 2013; Mollazade et al., 2013; Nejati-Koshki et al., 2013; Pourhassan-Moghaddam et al., 2013; Davaran et al., 2014; Kouhi et al., 2014; Wang et al., 2014)

\section{Acknowledgements}

The authors thank Department of Medical Nanotechnology, and Biotechnology Faculty of Advanced Medical Science of Tabriz University for all supports provided. This study was supported by a grant from Hematology and Oncology Research Center, Tabriz University of Medical Sciences.

\section{References}

Akbarzadeh A, Hosseininasab S, Davaran S, et al (2014). Synthesis, characterization, and In vitro studies of PLGAPEG nanoparticles for oral Insulin delivery. Chem Biol Drug Des, 3, 1-9.

Akbarzadeh A, Mikaeili H, Zarghami N, et al (2012). Preparation and in-vitro evaluation of doxorubicin-loaded $\mathrm{Fe}_{3} \mathrm{O}_{4}$ magnetic nanoparticles modified with biocompatible copolymer. Int J Nanomedicine, 7, 1-16.

Akbarzadeh A, Nejati-Koshki K, Mahmoudi Soghrati M, et al (2013). In vitro studies of NIPAAM-MAA-VP copolymercoated magnetic nanoparticles for controlled anticancer drug release. JEAS, 3, 108-15.

Ahmadi A, Shirazi H, Pourbagher N, Akbarzadeh A, Omidfar K (2014). An electrochemical immunosensor for digoxin using core-shell gold coated magnetic nanoparticles as labels. Mol Biol Rep, 41, 1659-68.

Akbarzadeh A, Rezaei A, Nejati-Koshki K, et al (2014). Synthesis and physicochemical characterization of biodegradable star-shaped poly lactide-co-glycolide- $\beta$ -cyclodextrin copolymer nanoparticles containing albumin, $J$ Adv Nanoparticles, 3, 1-9.

Akbarzadeh A, Rezaei-Sadabady R, Zarghami N, et al (2013). Studies of the relationship between structure and antioxidant activity in interesting systems, including tyrosol, hydroxytyrosol derivatives indicated by quantum chemical calculations. Soft, 2, 13-8.

Akbarzadeh A, Samiei M, Davaran S (2012). Magnetic nanoparticles: preparation, physical properties, and applications in biomedicine. Nanoscale Res Lett, 7, 14-26.

Akbarzadeh A, Samiei M, Joo SW, (2012). Synthesis, characterization and In vitro studies of doxorubicin-loaded magnetic nanoparticles grafted to smart copolymers on A549 lung cancer cell line. J Nanobiotechnol, 10, 46-52.
Akbarzadeh A, Zarghami N, Mikaeili H, et al (2012). Synthesis, characterization and In vitro evaluation of novel polymercoated magnetic nanoparticles for controlled delivery of doxorubicin. Nanotechnol Sci Appl, 5, 13-25.

Ebrahimnezhad Z, Zarghami N, Keyhani M, et al (2013). Inhibition of hTERT gene expression by silibinin-loaded PLGA-PEG-Fe3O4 in T47D breast cancer cell line. Bioimpacts, 3, 67-74.

Bagatell R, Whitesell L (2004). Altered Hsp90 function in cancer: a unique therapeutic opportunity. Molecular Cancer Therapeutics, 3, 1021-30.

Challa R, Ahuja A, Ali J, Khar R (2005). Cyclodextrins in drug delivery: an updated review. Aaps Pharmscitech, 6, 329-57.

Davaran S (2014). Physicochemical characteristics of Fe. Asian Pac J Cancer Prev, 15, 49-54.

Fukuyo Y, Hunt CR, Horikoshi N (2010). Geldanamycin and its anti-cancer activities. Cancer Lett, 290, 24-35.

Ge QD, Lv N, Kong YN, et al (2012). Clinical characteristics and survival analysis of breast cancer molecular subtypes with hepatic metastases. Asian Pac J Cancer Prev, 13, 5081-6.

Ghasemali S, Nejati-Koshki K, Tafsiri E, et al (2013). Inhibitory effects of -cyclodextrin-helenalin complexes on H-TERT gene expression in the T47D breast cancer cell line-results of real time quantitative PCR. Asian Pac J Cancer Prev, 14, 6949-53.

Guo W, Siegel D, Ross D (2008). Stability of the Hsp90 inhibitor 17AAG hydroquinone and prevention of metal catalyzed oxidation. J Pharm Sci, 97, 5147-57.

Karkoulis PK, Stravopodis DJ, Margaritis LH, Voutsinas GE (2010). 17-Allylamino-17-demethoxygeldanamycin induces downregulation of critical Hsp90 protein clients and results in cell cycle arrest and apoptosis of human urinary bladder cancer cells. BMC Cancer, 10, 481.

Kouhi M, Vahedi A, Akbarzadeh A, et al (2014). Investigation of quadratic electro-optic effects and electro-absorption process in GaN/AlGaN spherical quantum dot. Nanoscale Res Lett, 9, 1-6.

Mirakabad FT, Akbarzadeh A, Zarghami N, et al (2013). PLGAbased nanoparticles as cancer drug delivery systems. In: 1st Tabriz International Life Science Conference and 12th Iran Biophysical Chemistry Conference, Tabriz university of medical sciences.

Mollazade M, Nejati-Koshki K, Akbarzadeh A, et al (2013). PAMAM dendrimers augment inhibitory effect of curcumin on cancer cell proliferation: possible inhibition of telomerase. Asian Pac J Cancer Prev, 14, 6925-8.

Najafi B, Anvari S, Roshan ZA, et al (2013). Disease free survival among molecular subtypes of early stage breast cancer between 2001 and 2010 in Iran. Asian Pac J Cancer Prev, 14, 5811-6.

Nejati-Koshki K, Akbarzadeh A, Pourhasan-Moghaddam M, et al (2013). Inhibition of leptin and leptin receptor gene expression by silibinin-curcumin combination. Asian Pac J Cancer Prev, 14, 6595-9.

Pick E, Kluger Y, Giltnane JM, et al (2007). High HSP90 expression is associated with decreased survival in breast cancer. Cancer research, 67, 2932-7.

Pourhassan-Moghaddam M, Rahmati-Yamchi M,Akbarzadeh A, et al (2013). Protein detection through different platforms of immuno-loop-mediated isothermal amplification. Nanoscale research letters, $8,1-11$.

Richardson PG, Mitsiades CS, Laubach JP, et al (2011). Inhibition of heat shock protein 90 (HSP90) as a therapeutic strategy for the treatment of myeloma and other cancers. $\mathrm{Br}$ J haematol, 152, 367-79.

Sakthivel K, Kannan N, Angeline A, Guruvayoorappan C (2012). Anticancer activity of acacia nilotica (L.) Wild. Ex. Delile 
subsp. indica against Dalton's ascitic lymphoma induced solid and ascitic tumor model. Asian Pac J Cancer Prev, 13, 3989-95.

Schulz R, Marchenko ND, Holembowski L, et al (2012). Inhibiting the HSP90 chaperone destabilizes macrophage migration inhibitory factor and thereby inhibits breast tumor progression. J Exp Med, 209, 275-89.

Tsuda H (2010). Risk assessment studies of nanomaterials in Japan and other countries. Asian Pac J Cancer Prev 11

Usmani SZ and Chiosis G (2011). HSP90 inhibitors as therapy for multiple myeloma. Clin Lymphoma Myeloma Leuk, 11, 77-81.

Valizadeh A, Mikaeili H, Samiei M, et al (2012). Quantum dots: synthesis, bioapplications, and toxicity. Nanoscale Res Lett, 7, 1-14.

Wang H-H, Song YX, Bai M, et al (2014). Ultrasound targeted microbubble destruction for novel dual targeting of hsp72 and hsc70 in prostate cancer. Asian Pac J Cancer Prev: APJCP, 15, 1285.

Yadav D, Anwar MF, Garg V, et al (2014). Development of polymeric nanopaclitaxel and comparison with free paclitaxel for effects on cell proliferation of MCF-7 and B16F0 carcinoma cells. Asian Pac J Cancer Prev, 15, 2335-40.

Yallapu MM, Jaggi M, Chauhan SC, et al (2012). Curcumin nanoformulations: a future nanomedicine for cancer. Drug Discovery Today. 17, 71-80.

Yin H-T, Zhang D, Wu X, et al (2013). In vivo evaluation of curcumin-loaded nanoparticles in a A549 xenograft mice model. Asian Pac J Cancer Prev, 14, 409-12.

Zajac M, Gomez G, Benitez J, et al (2010). Molecular signature of response and potential pathways related to resistance to the HSP90 inhibitor, 17AAG, in breast cancer. BMC Medical Genomics, 3, 44. 